\title{
Design of Medical Assistant Robot
}

\author{
Min Ding \\ School of North China Electric Power University, North China Electric Power University, \\ Baoding 071000, China. \\ dingmin1995@foxmail.com
}

\begin{abstract}
Keywords: Biomedical Engineering; Medical assistant robot; Sensor.
\end{abstract}
\begin{abstract}
Research on medical assistant robot makes Biomedical Engineering Experiment get rid of the dependence on training special operations personnel to complete a high efficiency, high success rate and high difficulty of the operation. It could be a revolutionary change in the means of biomedical engineering experiments. Our primary goal in this study is to design a small wheeled robot. This robot is controlled by STM32 microcontroller as the core of medical service robot. It is combined with laser sensors, infrared sensors, eight-channel grayscale sensors and flexible synchronous wheels and manipulator which are spliced by JUN YUAN. The robot can automatically complete a series of functions such as: bypass surgery, stent procedures and other rules of the medical task. Then it can quickly return to the starting area. The innovation of this medical assistant robot is that its chassis is made of ACRYLIC which makes our robot get rid of the traditional clumsy and heavy on chassis of robot. Apart from that flexible robot hyperactivity let crawl, place objects more diverse. In addition, the using of sensing elements makes our robot more perfect, having an absolute advantage both in speed and accuracy. The study not only allows us to have a deeper understanding on biomedical engineering and the working principle of robots, but also improves our ability to become calm and smart.
\end{abstract}

\section{Introduction}

Biomedical engineering was founded in 1950 s, which has a very close relationship with medical engineering and biotechnology. And now the biomedical engineering has developed pretty rapidly and become one of the main areas of competition in the world. Biomedical engineering ---- the noun first appeared in the United States. Like other disciplines, the development of biomedical engineering is determined by the factors of science, technology, society and economy. In addition to extremely good social benefits, but also good economic benefits, the prospect of the biomedical engineering is very broad. In 1984, for example, the market scale of the U.S. biomedical engineering was about $\$ 11$ billion. American Academy of Science estimated that by 2000 its output was expected to reach $\$ 400$ to 100 billion.

In combination with medical conditions, the biomedical engineering is developed on the basis of electronics, microelectronics, modern computer technology, chemistry, polymer chemistry, mechanics, physics, optics, X-ray technology, precision machinery and modern high technology. Its development process is closely related to the development of high technology in the world, at the same time it uses almost all of the high-tech achievements, such as aerospace technology, microelectronics technology and so forth.

The research on medical assistant robot helps the biomedical engineering experiment get rid of training workers with special skills and achieve the high efficiency and high success rate, high difficulty operation at the same time.

In order to reduce the workload of medical staff, we have designed a new medical assistant robot. As shown in the figure 1 and figure 2, this kind of medical assistant robots is a small wheeled robot, which is controlled by STM32 microcontroller, combining with laser sensors, infrared sensors, gray-scale sensors of eight channels. The robot can play a great important role in helping the doctors and nurses complete both bypass surgery and stent surgery since it has flexible synchronous wheels and mechanical hands. 


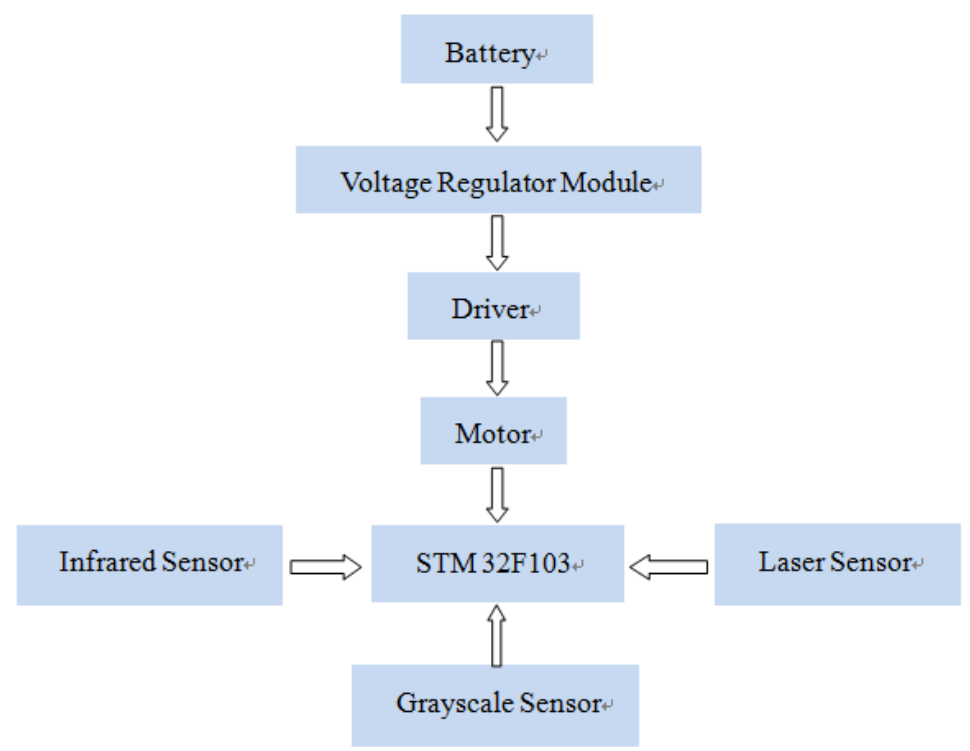

Fig. 1 integrated system design

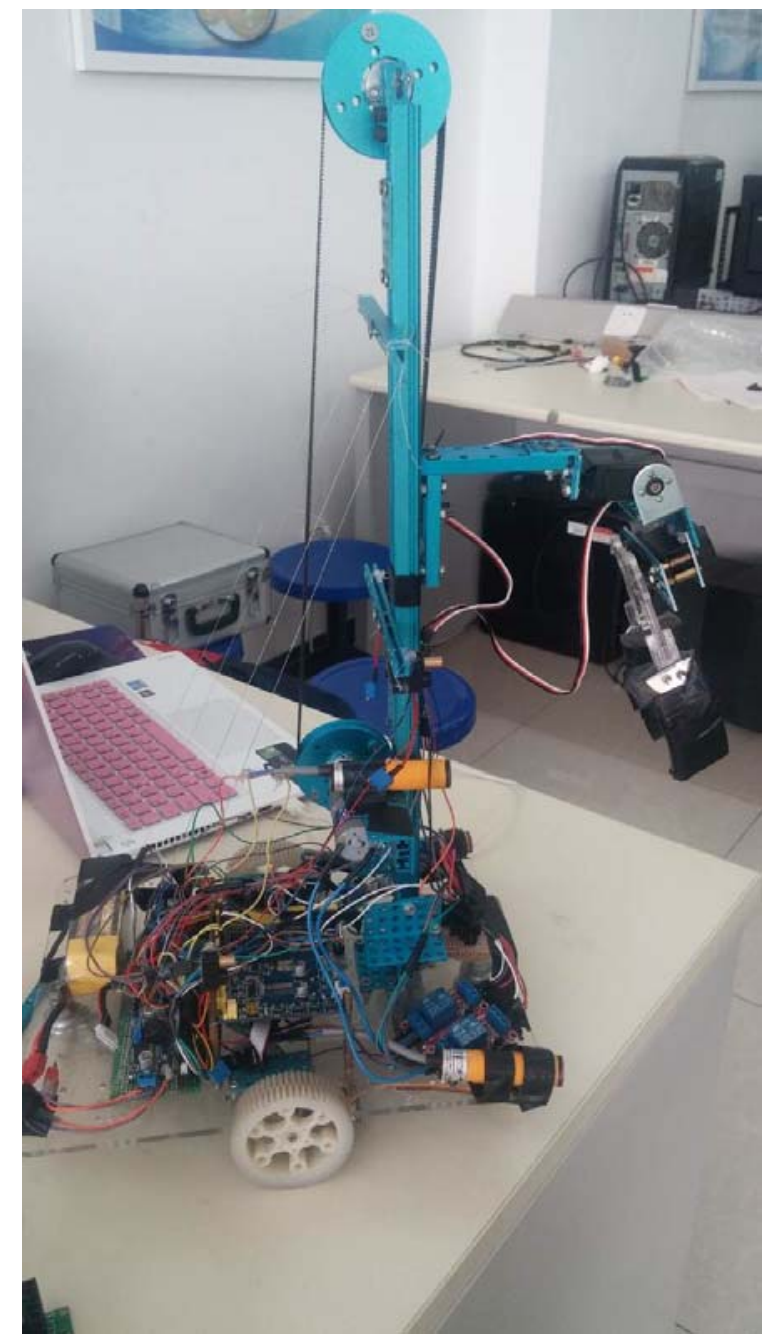

Fig.2 picture of real products

From the aspect of movement, our robot has been improved on the basis of classic wheeled mobile ---- two driving wheels and two universal wheels can make the robot car rotate in the horizontal plane at different speeds so that the medical assistant robot can turn a corner with no radius and move in 360 degrees of the forward direction, breaking the single way forward. 
The front and rear of the vehicle body are respectively provided with two grayscale sensors of eight channels. Gray scale sensor is analog sensor, which uses the principle that the detection surface of different colors can reflect light in different degrees and then convert the intensity of the light to a signal that the robot can recognize. Through a decoder to realize data samples in turn, it can be very convenient for the medical assistant robot to track and monitor the linear line, the T-shaped line and also the cross lines. Infrared obstacle avoidance sensor is arranged in front of the vehicle body, which is essentially is a NPN type photoelectric switch. When an object is detected, the I/O port will output low level of 0 , while the high level 1 will be output in normal states. This method can determine the robot's walking state and the location of the object, then stop at the specified location and wait for the next instruction. Avalanche photodiode is an optical sensor with a magnifying function, so it can detect extremely weak light signal and convert it to the corresponding electrical signal. We use the laser sensor so that the robot can accurately locate and place the object on the platform of the bypass surgery and stent surgery, completing the task successfully.

\section{Summary}

To sum up, our medical assistant robots defeat the traditional robots which can only track relying on the identification of ground fixed line. The new robot can forward without any markings on the road, only relying on laser sensor to avoid obstacle and complete the corresponding mobile task. On the other hand, we use the steering gear to control the flexible manipulator. By installing the steering gear at different locations and also with the help of Lifting of synchronous wheels, the object can be taken up and placed sideways flexibility at different height. In order to solve the problem that the center of gravity is too high for the robot to move, we also use the stability theory of triangle to transform it, and make it more suitable for the practical engineering.

\section{Acknowledgments}

My deepest gratitude goes first and foremost to Processor Fang, my supervisor, for her constant encouragement and guidance. She has walked me through all the stages of the writing of the thesis. Without her consistence and illuminating instruction, this thesis could not have received its present form.

Last, I would like to express my heartfelt gratitude to my partner $\mathrm{Hu}$ and $\mathrm{Lu}$, who helped me a lot in this project.

\section{References}

[1] Xizheng Zhang, The research and development of medical robot, Medical equipment in China, 2009

[2] Huoliang Liu, The guide of exploiting STM32, China Machine Press, 2013-07-01

[3] Xiuning Chen, Fundamentals of mechanical design, Zhejiang University Press, 2007-07-01 